\title{
UM BREVE RELATO DA "1988 BABSON COLLEGE ENTREPRENEURSHIP RESEARCH CONFERENCE"
}

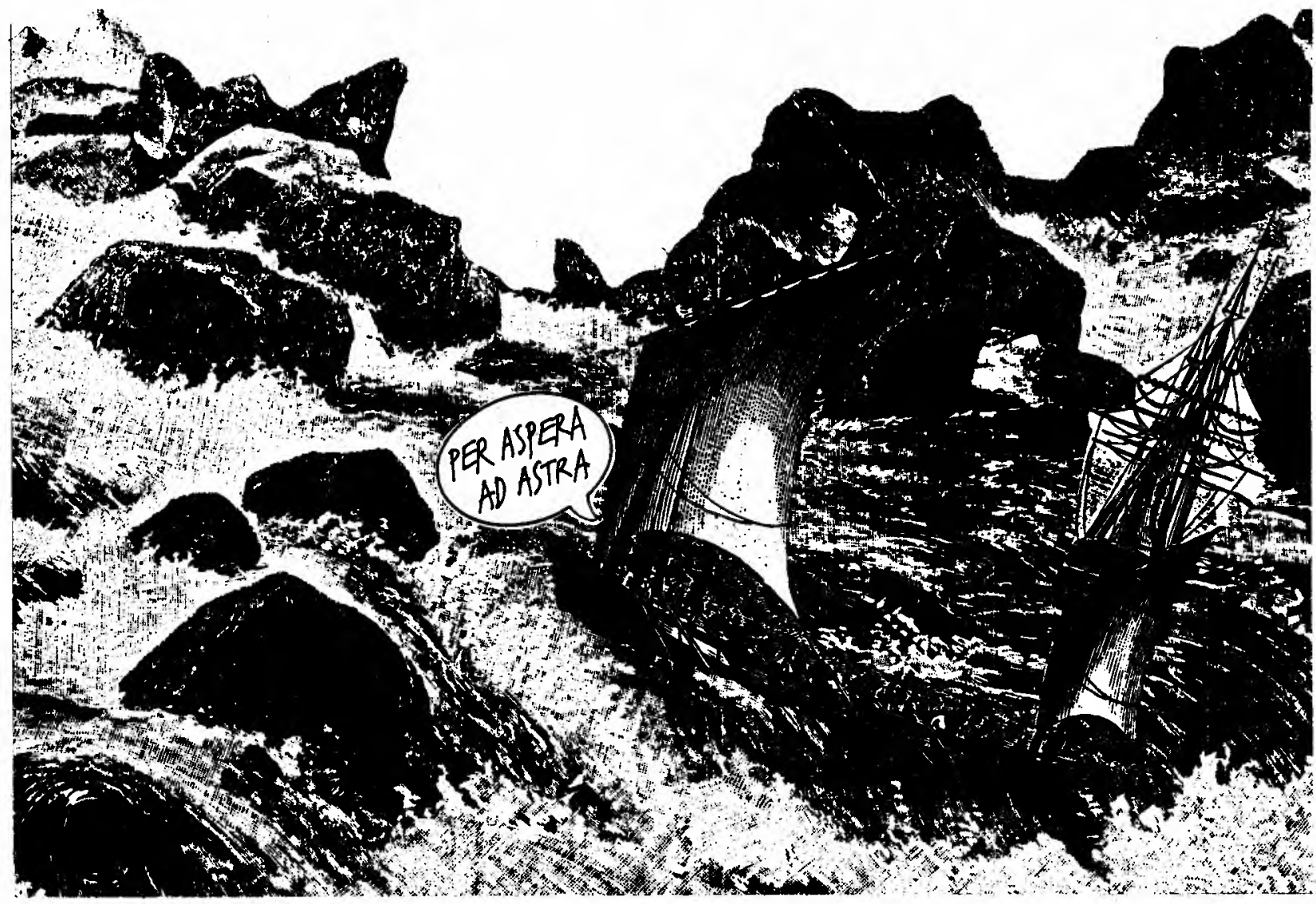

\section{- MARCElo alCEU AMOROSO LIMA}

Professor Assistente no Departamento de Engenharia de Produçăo da Universidado Federal de Săo Carlos.

\section{INTRODUCÃO}

A conferências do Babson College sobre No$A$ vos Negócios " começaram em 1981, com 39 trabalhos apresentados, e desde então ocorre- ram anualmente, até esta última, realizada em maio de 1988 em Calgary, Canadá, onde foram apresentados 106 papers em 36 seçठes de trabalho, com 185 autores e co-autores. Em oito anos desta Conferência, cerca de 430 pesquisadores apresentaram trabalhos. Ressalte-se ainda que 0

(") Neste texto, os termos entrepreneurship e entrepreneurs foram traduzidos, respectivamente, por novos negócios e empreendedores. 
indice de rejeição de trabalhos submetidos em 88 foi de $50 \%$ e que $30 \%$ dos trabalhos apresentados na Conferência deverão ser publicados na Revista Frontiers of Entrepreneurship.

A "Babson Conference" é o evento acadêmico mais prestigiado a nivel mundial na área, sendo outros eventos relevantes o encontro anual do "International Council for Small Business" e a "Annual International Conference on University Related Research Parks". A reunião anual da Ácademy of Management, que é a principal reunião acadêmica em Administração de Empresas nos E.U.A., vem também, pouco a pouco, absorvendo alguns trabalhos.

Dada a importância da Babson Conference, faremos a seguir um breve relato do conteúdo dos trabalhos ali apresentados que nos pareceram mais relevantes. $O$ objetivo foi oferecer uma seleção prévia de informações aos leitores que não estiveram presentes a esta Conferência, de modo que possam fazer sua própria triagem e depois consultar os textos que sejam de seu interesse específico. Em sua grande maioria, os trabalhos apresentados contiveram métodos estatísticos como cluster analysis, factor analysis etc., aplicados em surveys realizadas com o objetivo de testar determinadas hipóteses.

\section{II-PRINCIPAIS RESULTADOS DOS TRABALHOS APRESENTADOS NA CONFERÊNCIA}

Os trabalhos apresentados podem ser agrupados a partir de cinco grandes enfoques:

1. Caracterização de novos negócios (sturt up) e de seus empreendedores e gerentes ( $45 \%$ dos trabalhos).

2. Características e performance das instituiçð̃es financiadoras, indutoras e apoiadoras da criação de novos negócios (25\% dos trabalhos).

3. Estratégia de crescimento dos novos negócios e a dinâmica competitiva dos mercados (10\% dos trabalhos).

4. Características dos novos negócios dentro de grandes corporações (cerca de $10 \%$ dos trabalhos).

5. Novos negócios e desenvolvimento econômico e social (cerca de $10 \%$ dos trabalhos).

II.1. Caracterização de novos negócios (start up) e dos seus empreendedores e gerentes ( $45 \%$ dos trabalhos).

Quanto ao primeiro enfoque, pode-se subdividi-lo entre trabalhos preocupados com a análise das características dos novos negócios e dos seus empreendedores $(50 \%)$, trabalhos que analisam as diferenças entre os empreendedores de distintas culturas ou oriundos das "minorias" étnicas e sociais e trabalhos que analisam as diferenças entre empreendedores $e$ gerentes.

Com relação às características principais dos empreendedores, emergem seis grandes fatores motivacionais para a criação do novo negócio, de acordo com uma pesquisa de Scheinberg (1) em onze países: busca de reconhecimento social, valorizaçăo do enriquecimento, busca de contribuir com a comunidade, busca de um desenvolvimento pessoal/profissional, busca de independência, insatisfação com o trabalho anterior; ou então, de acordo com Dubini (2), três grandes grupos de motivação podem ser apontados: os empreendedores auto-realizadores que começam seus negócios impulsionados por um desejo de realização, de independência e autonomia, os empreendedores descontentes que começam seus negócios pela insatisfação com as condições de trabalho anteriores e os empreendedores que seguem uma tradição de família.

Uma observação interessante é, de acordo com Sverker (3), que a importancia motivacional varia de acordo com diferentes culturas; outra é que, ao invés de incentivos gerais aplicados a uma vasta área geográfica, políticas governamentais específicas a uma determinada realidade regional e contextual parecem ser mais efetivas ${ }^{(4)}$.

O trabalho de Gillin ${ }^{(5)}$ aponta, para a Austrália e pela ordem, os seguintes fatores de

1. SCHEINBERG, Sari (New York University) \& LAURENCE, William J. (Pace University). The Economic, Political and Cultural Environment and Its Effect on Entrepreneurial Motioation.

2. DUBINI, Paola (Universitá Commerciale L. Bocconi - SDA Bocconi) \& MacMILLAN, Ian C. (University of Pennsylvania). Entrepreneurial Prerequisites in Venture Capital Backed Projects.

3. ALANGE, Sverker (Chalmers University) \& SCHEINBERG, Sari (New York University). Swedish Entrepreneurship in a Cross-Cultural Perspective.

4. DUBINI, Paola (Universitá Commerciale L. Bocconi - SDA Bocconi). Motivational and Environmental Influences on Business Start-Ups: Some Hints for Public Policies.

5. GILLIN, Murray L. (Swinburne Institute of Technology) \& HINDLE Kevin (Kevin Hindle Management Insight). Some Key Success Factors in Australian New Venture Management. 
sucesso de um novo negócio na fase de evolução entre o início do empreendimento e a evidência do sucesso comercial:

a) fatores de marketing;

b) fatores de gerenciamento;

c) fatores de integração;

d) fatores tecnológicos;

e) fatores financeiros;

f) fatores de assistência governamental.

O trabalho de Hills ${ }^{(6)}$, por sua vez, também analisa fatores de sucesso em novos negócios com alta taxa de crescimento e chega aos seguintes resultados, pela ordem:

a) conhecimento do produto, do mercado e da indústria;

b) flexibilidade e adaptabilidade à mudança;

c) persistência, dedicaçăo e "trabalho duro";

d) exploração de um nicho de mercado.

II.2. Característica e performance das instituições financiadoras, indutoras e apoiadoras da criação de novos negócios (25\% dos trabalhos).

Com relação ao segundo enfoque, as instituiçס̄es analisadas são basicamente as empresas de capital de risco (venture capital), as iniciativas de apoio do governo e da comunidade e as instituições de ensino, pesquisa e consultoria envolvidas com o assunto.

Boberg (7), analisando os cursos e programas existentes sobre novos negócios na Europa e nos Estados Unidos, constata um crescimento de dez vezes, desde 1958; constata também uma tendência dos cursos de administração de darem uma ênfase crescente a esta área, de certa forma substituindo o enfoque de administração de pequenas empresas por este, de novos negócios, mais dinâmico. Scott ${ }^{(8)}$, analisando o Programa para Estudantes Empreendedores, implantado no Reino Unido, constata a necessidade de atividades de propaganda da "opção de criação de um novo negócio" como uma perspectiva profissional viável, bem como a necessidade de uma formação mais prática nas disciplinas voltadas para novos negócios. Stevenson (9) argumenta no sentido da interdisciplinaridade do ensino e da pesquisa de novos negócios, em termos de que seu estudo "é uma atividade integrativa, baseada na capacidade de compreender dilemas muito complexos, no que diz respeito a próposito, possibilidades e ferramentas".

Klofsten (10), comparando spin offs de universidades com spin offs de empresas em Linköping, Suécia, quanto à importância e disponibilidade de recursos internos e externos para o desenvolvimento do novo negócio, constata que ambos os grupos tiveram dificuldades iniciais no aporte do capital necessário, embora o primeiro grupo tenha tido mais. O grupo universitário teve mais dificuldade com o conhecimento do mercado e o das empresas com a obtenção da mão de obra qualificada necessária. Como um todo, os spin offs das universidades tendem a ter uma estrutura inicial menos completa e balanceada que a das empresas.

Freear (11), estudando as fontes de capital para novos negócios com base tecnológica, na região da Nova Inglaterra, nos E.U.A., constata o papel complementar exercido pelas empresas de capital de risco (venture capital) e pelos capitalistas individuais, em que aquelas investem quantias maiores e num estágio posterior, e estes investem quantias menores em estágios menos desenvolvidos do negócio.

Doutriaux (12), examinando as características de empresas de alta tecnologia no Canadá que tiveram seu início baseado em encomendas governamentais (indústrias de microeletrônica e comunicaçōes), confirma o papel fundamental das encomendas do governo para o crescimento destas empresas e para o desenvolvimento tec-

6. HILLS, Gerald E. (University of Illinois at Chicago) \& WELSH, Harold P. (De Paul University). High Growth Entrepreneurial Venture: A Content Analysis to Identify Common Strategic Factors.

7. BOBERG, Alice (University of Calgary) \& KEEKER, Pamela (University of Calgary). Changing Patterns of Demand: Entrepreneurship Education for Entrepreneurs.

8. SCOTT, Michael G. (University of Stirling). Aspects of the Long Term Supply of Entrepreneurs: The $U$. K. Experience of Encouraging Graduate Enterprise.

9. STEVENSON, Howard H. (Harvard University). General Management and Entrepreneurship.

10. KLOFSTEN, Magnus (Linköping University); LINDELL, Pia (Linköping University); OLOFSSON, Christer (Linköping University) \& WAHLBIN, Clas (Linköping University). Internal and External Resources in Technology-Based Spin-Offs: a Survey.

11. FREEAR, John (University of New Hampshire) \& WETZEL, William E. (Babson College). Equity Financing for New Technology-Based Firms.

12. DOUTRIAUX, Jerome A. (University of Ottawa). Government Procurement and Research Contracts at Start-Up and Success of Canadian High-Tech Entrepreneurial Firms. 
nológico do setor.

II.3. Estratégia de crescimento dos novos negócios e a dinâmica competitiva dos mercados (10\% dos trabalhos).

Com relação ao terceiro enfoque, uma série de trabalhos com contribuiçōes originais foram apresentados.

McDougall (13) identificou oito padrões de estratégia de novos negócios em diferentes indústrias:

a) crescimento agressivo através da venda de produtos do tipo commodity para numerosos mercados, através de pequenas ordens dos clientes;

b) crescimento agressivo através de novos produtos, competitivos em preço, para grandes clientes;

c) crescimento agressivo através de produtos especiais e específicos com preços competitivos e para alguns grandes clientes;

d) crescimento moderado, através de uma vasta gama de produtos para muitos mercados e uma grande integração "para trás";

e) crescimento controlado, através de produtos com um preço "extra" vendidos diretamente ao consumidor;

f) crescimento limitado, em pequenos nichos de mercado, oferecendo um produto superior em performance e um bom serviço "pósvenda";

g) crescimento moderado, através de constante desenvolvimento de novos canais de fixação da marca do produto e grande promoção de vendas;

h) crescimento limitado através de produtos com vendas esporádicas para muitos mercados e com alguma integração "para frente".

McDougall faz a importante constatação de que a grande "chave" para explicar a performance dos novos negócios é a interação da estrutura industrial com a estratégia empresarial, ou seja a necessidade de uma integração da área de conhecimento de Estratégia Empresarial com a área de conhecimento de Organização Industrial.

Covin ${ }^{(14)}$ estudou cerca de 20 novos negócios, procurando observar e contrastar as estratégias empresariais em indústrias emergentes, em crescimento e maduras, concluindo que há uma performance relativamente melhor dos novos negócios em indústrias em crescimento, seguidas pelos novos negócios em indústrias maduras e, por fim, pelos novos negócios em indústrias emergentes.
De acordo com Covin, em relação "às firmas $\mathrm{em}$ indústrias em crescimento, as firmas em indústrias emergentes dependiam em geral mais de: a) financiamentos externos; $b$ ) direitos autorais e patentes de produtos e processos. Relativamente às indústrias maduras, as firmas em indústrias emergentes tendiam mais a: a) financiamentos externos; b) ter preços de produtos/serviços altos; c) ter uma menor gama de produtos; d) enfatizar o desenvolvimento de novos produtos e serviços; e) depender de patentes ou direitos autorais de produtos e processos; $f$ ) usar técnicas e tecnologias operacionais novas; g) enfatizar redução de custo, produtividade dos empregados e eficiência operacional; $h$ ) aceitar dependência de um único fornecedor ou consumidor; i) tentar ativamente predizer os requisitos $e$ movimento dos concorrentes $e$ tendências da indústria. Finalmente, em relação às firmas das indústrias maduras, as firmas das indústrias em crescimento tendiam mais a: a) usar propaganda; b) enfatizar o desenvolvimento de novos produtos e serviços; c) usar tecnologias e técnicas operacionais inovadoras; d) aceitar a dependência de um único consumidor ou fornecedor."

Como conclusão geral, Covin observa que os resultados de sua pesquisa sugerem que os novos negócios nos três tipos de indústria se engajam em estratégias competitivas que são, em larga medida, específicas a cada tipo.

Duchesneau (15), estudando 26 novos negócios (13 sucessos e 13 fracassos) da indústria de distribuição de suco de laranja natural nos E.U.A.,

13. McDOUGALL, Patricia P. (Georgia State University) \& ROBINSON, Richard B. (University of South Carolina). New Venture Performance: Patterns of Strategic Behavior in Different Industries.

14. COVIN, Jeffry G. (Georgia Institute of Technology) \& SLEVIN, Dennis P. (University of Pittsburgh). The Strategic Management of New Ventures in Emerging, Growing, and Mature Industries.

15. DUCHESNEAU, Donald A. (Fresh Citrus Products, Inc.) \& GARTNER William B. (Georgetown University). A profile of New Venture Success and Failure In An Emerging Industry. 
uma indústria hoje emergente, homogênea e composta de pequenas empresas, procurou explorar três dimensões de variáveis que poderiam diferenciar os novos negócios bemsucedidos dos malsucedidos:

a) as características do principal empresário;

b) o padrão de início do novo negócio;

c) o comportamento da empresa no que diz respeito à sua organização e às práticas da indústria.

O perfil das empresas bem-sucedidas revelou que os principais empresários tinham nivel educacional, experiências empresariais anteriores, conhecimento gerencial diversificado, capacidade de comunicação, autoconfiança, independência e cautela em nível mais elevado que os empresários malsucedidos. As empresas bem-sucedidas eram, em geral, aquelas iniciadas com metas ambiciosas, com uma idéia ampla e clara do negócio e com uma grande dedicação e perseverança por parte do empresário. As jointventures foram os formatos organizacionais mais adaptados às mudanças da indústria e as empresas mais bem-sucedidas foram as organizações mais flexíveis e participativas. Níveis maiores de investimento inicial estiveram claramente associados ao sucesso das empresas, assim como as empresas bem-sucedidas foram as que procuraram crescer e vender para amplos setores do mercado. Embora o serviço ao consumidor tenha sido uma preocupação importante, o caráter de commodity do suco natural impediu a existência desse tipo de diferenciação e as firmas que tiveram por foco estes serviços acabaram tendo custos altos e se inviabilizaram.

Phillips (16), preocupado com o lugar comum de que "quatro de cinco empresas criadas vão à falência nos cinco primeiros anos", ou seja, uma taxa de sobrevivência de $20 \%$, procurou fazer uma análise empírica da dinâmica da criação de pequenas empresas e de seu crescimento inicial nos Estados Unidos. Sua primeira constaţação é que, na verdade, duas de cada cinco empresas sobrevivem pelo menos seis anos (ou seja, uma taxa de sobrevivência de $40 \%$ ) e mais do que a metade das sobreviventes crescem. Uma segunda e fundamental constatação é que as empresas que conseguem sobreviver, conseguem em geral crescer; isto é, firmas com uma taxa de crescimento média ou alta, apresentaram uma taxa de sobrevivência de $75 \%$. Phillips considera que a taxa de crescimento é o fator que mais influencia a sobrevivência. O padrão típico de crescimento de uma pequena empresa corresponde à teoria do ciclo de vida: há um período inicial de crescimento pequeno e muito esforço, seguido de um grande salto no crescimento, na altura do sexto ano. Isto sugere que a maioria das empresas que sobrevivem por quatro ou cinco anos, provavelmente alcança esta etapa de crescimento, e por causa dela sobrevive.

Como era esperado, houve uma grande variação da taxa de sobrevivência entre indústrias, cuja explicação esteve associada basicamente às suas diferentes taxas de crescimento.

Cooper (17), observando dados de $3000 \mathrm{em}-$ presários com negócios iniciados nos últimos 17 meses nos E.U.A., constatou uma taxa de falência de apenas $10 \%$ a.a., ao acompanhar estas cerca de 3000 empresas durante dois anos. Os contrastes entre sobreviventes e malsucedidos foram então analisados, notando-se diferenças nas seguintes dimensões:

a) educação do empreendedor;

b) experiência na indústria;

c) tamanho inicial da empresa.

Surpreendentemente para Cooper, outras variáveis como nível anterior de experiência gerencial e propriedade prévia de um negócio não estiveram relacionadas à sobrevivência.

Miller (18), ao observar o elevado número de trabalhos existentes sobre o padrão de comportamento dos empreendedores e a ausência de pesquisa sobre as estratégias existentes ou mais adequadas, com relação aos novos negócios, fez uma interessante revisão bibliográfica do "estado da arte" e propôs algumas questões fundamentais para avançar a fronteira do conhecimento. De acordo com suas palavras,

"na literatura revisada, três dimensões de Estratégias de Entrada devem ser considera.ias: Timing, Agressão e Vantagem Competitiva. $O$ Timing da entrada é discutido em termos da ordem de entrada. A agressão é vista como um conceito multidimensional, envolvendo promoção e propaganda, alvos de mercado, e inves-

16. PHILLIPS, Bruce D. (U. S. Small Business Administration) \& KIRCHHOFF, Bruce A. (Babson College).An analysis of New Firm Survival and Growth.

17. COOPER, Arnold C. (Purdue University); DUNKELBERG, William C. (Temple University) \& WOO, Carolyn Y. (Purdu University). Survival and Failure: a Longitudinal Study.

18. MILLER, Alex (University of Tennessee). Entry Strategy and Its Relation to Venture Performance and Competitor Response: A Literature Review and Proposed Research Agenda. 
timento em capacidade de produção. Vantagem Competitiva é considerada uma função do posicionamento do produto em termos de diferenciação e liderança de custo.

Estes elementos estão relacionados a dois objetivos estratégicos para novos negócios. Um $e ́$ de maximização de suas performances em termos de posicionamento de mercado (e.g. fatia de mercado) e retorno financeiro (e.g. retorno sobre o investimento). O outro é a minimização das respostas competitivas das empresas existentes aos novos negócios que inoadiram 'seu' mercado.

As pesquisas sugerem que as estratégias que são desejáveis em termos de posicionamento no mercado e objetivos de rentabilidade podem ser indesejáveis em termos das respostas suscitadas nos competidores pré-existentes. Sugestōes específicas para questões a pesquisar que clarificariam o conhecimento desta área são:

a) as respostas competitivas das empresas préexistentes estão negativamente relacionadas ao posicionamento no mercado $e$ à performance financeira dos novos concorrentes? Em outros termos, as respostas competitivas são efetivas?

b) o Timing de entrada está relacionado ao posicionamento de mercado e à performance financeira, mas estará também relacionado à resposta competitiva?

c) as relaçôes entre os atributos estratégicos de Agressão e Vantagem Competitiva $e$ as variáveis de performance em termos de fatia de mercado e rentabilidade são moderadas pela intensidade tecnológica?"

II.4. Características dos novos negócios dentro de grandes corporaçðes

(cerca de $10 \%$ dos trabalhos).

Com relação ao quarto enfoque, Knight (19), analisando 133 novos negócios nos E.U.A. que se formaram a partir de spin offs de grandes empresas que montaram novos negócios para si, observou que $60 \%$ destes foram montados na área de sua experiência de trabalho anterior, enquanto $40 \%$ foram montados em outras áreas. Na média, os spin offs trabalharam cerca de dez anos em grandes corporações e começaram seus novos negócios com 32 anos. Knight também constata no trabalho que existiriam várias maneiras, em geral ignoradas, de as corporações aproveitarem a capacidade empresarial dos spin offs, principalmente através de joint ventures.

II. 5. Novos negócios e desenvolvimento econômico e social (cerca de $10 \%$ dos trabalhos).

Os trabalhos apresentados nesta área descrevem estudos de caso sobre os benefícios da criação de novos negócios em determinadas áreas ou regiões, urbanas ou rurais.

\section{QUESTÕES IMPORTANTES ADVINDAS DA CONFERÊNCIA, PARA O BRASIL E PARA AS NOVAS EMPRESAS DE ALTA TECNOLOGIA}

É interessante resgatar a apresentação de Bygrave (20) ao Doctoral Consortium, uma reunião de postulantes ao doutorado na área de novos negócios que antecedeu à Conferência, onde este autor aborda questões metodológicas referentes ao desenvolvimento desta área, constatando que o caráter recente do paradigma existente requer mais trabalhos de campo, estudos de caso em profundidade e pesquisa empírica, em relação a modelos teóricos ou à utilização de métodos estatísticos sofisticados.

No Brasil, dada a quase completa ausência de trabalhos e pesquisadores na área, há que se fazer ainda muita pesquisa sobre os motivos e o contexto em que se iniciam os novos negócios; em particular, a nosso ver, em indústrias com alto potencial de desenvolvimento e crescimento, como é o caso do setor de alta tecnologia. Neste caso, quer-nos parecer de extrema importância a abordagem de McDougall (21), já citada, da essencialidade de se estudar a interação da estrutura industrial com a estratégia empresarial, ao que nos permitiríamos, no caso do Brasil, acrescer o contexto institucional.

Outro ponto relevante parece ser a importância de se estimularem, no Brasil, as experiências citadas por Scott (22) de ensino de novos negócios para estudantes universitários em geral, como forma de promover a busca de novas alternativas de empreendimentos, em particular em áreas tecnológicas.

Enfim, esta é, sem dúvida, uma área de fundamental importância para induzir o desenvolvimento do país de uma forma mais criativa, mais rica e mais intensa e incorporando mais valor aos recursos naturais existentes.

19. KNIGHT, Russell M. (The University of Western Ontario). Spinoff Entrepreneurs: How Corporations Really Create Entrepreneurs.

20. BYGRAVE, William D. (Babson College). Research Methodology and Entrepreneurship. Apresentado ao Doctoral Consortium of the Conference.

21. Ver nota 13.

22. Ver nota 8 . 